\title{
PARTICLES AND ENERGY TRANSPORT IN THE SOLAR ATMOSPHERE DURING SOLAR FLARES
}

HERISTCHI ', D., RAADU ${ }^{2}$, M.A., VIAL., J.-C. ${ }^{3}$, and MALHERBE, J.-M. ${ }^{\prime}$

1 : Observaloire de Paris, Section de Meudon, Dasop, U.A. 326, 92195 Meudon Principal Cedex, FRANCE

2 : Royal Institute of Technology, Department of Plasma Physics, S-100 44 Slockhoim, SWEDEN

3 : I.A.S./ Laboratoire de Physique Stellaire et Planétaire, B.P. 10, 91371 Verrières-le-Buisson Codex, FRANCE

\section{Abstract:}

The proposed model of particles transpont in the sular atmosphere during flares consisis in a low density plasmoid originating deep in the almosphore and rising under magnotic and buoyancy forces. Contined particles are selectively released during the ascont and thoir interaction with the solar atmosphere produces $X$ and $\gamma$ bremsstrahlung. The characteristics of high energy paricies released in the interplanelary medium are found 10 agree with observations.

\section{INTRODUCTION}

It is cenerally accepted that particles accelerated during solar flares interact with the solar atmosphere and escape and diffuse in the interplanetary medium (i.m.). Such interacting particles are belioved 10 he the primary source of energy in the flare process. In most models (Svestka 1976, Ramaty and Murphy 1987), particles are accelerated high in the atmosphere, and soms of them impinge on the low atmosphere while others an: ejuclud in the i.m. Wut such models fail 10 explain both properties of energetic particles recorded in the i.m. : chemical and isotopic composition and energy spectra. We discuss here the possibility of a very different model where particles are transported upwards in an isolated structure.

II. CHARACTERISTICS OF FLARES PARTICLES IN THE I. M.

We concentrate here on some major characteristics of energelic particles recorded in the i.m.

\section{Chemical and isotonic composition.}

The ratio 3 te $/$ the is far larger than the cosmic value and depends on the events with a range of $10^{4}$ (Kocharcv and Kocharov 1984). The same is true for Fe. There is also evidence for the existence of partially ionized atoms ( $\mathrm{He}^{+} \approx 10 \%$, Fan et al 1984, Hovestad et al 1931).

These two featurcs are hard to explain if acceleration occurs high in the atmosphere. 


\section{Enerax Spectra.}

Energy spectra of energetic particles recorded in the i.m. follow a power law that varies with time (as the result of scattering in the $i$. m.). The source spectra of particles are generally derived from the different energy-band maxirual intensities, whatever their times (Bryant et al 1964). These spectra must be corrected : for instance, for isotropic diffusion, we have :

$$
I=\frac{N v}{32 \pi(\pi \lambda v t / 3)^{1.6}} \exp \left(-\frac{3 r^{2}}{1 \lambda v t}\right)
$$

where $N$ is the total number of particles in a given energy band, $t$ the time, $v$ the velocity, $\lambda$ the mean free path, $r$ the distance from the source, and 1 the intensity).

With this formula, if $\lambda$ is the same for all energies, the above profiles are the same us functions of the travelled distance $v t$ within the factor $N v$. So the spectrum formed with maximal intensities must be corrected by a $1 / \mathrm{v}$ factor. Then the source spectra of the i.m. particles often follow a power law and indicate, in all cases, a small traversed material.

We conclude that in the i. $m$., energetic particles have the characteristics of both": the HIGH atmosphere (spectrum) and the LOW atmosphere (composition). Moreover, the very existence of partially ionized He requires a low origin on one hand, and the absence of any interaction wilh the atmosphere on the other hand.

\section{THE PROPOSED MODEL}

As a solution of the above problems, we propose a plasmoid (magnetically isolated structure) that originates deep in the solar atmosphere and rises under magnetic and buoyancy forces (Heristchi et al 1988 and Figure 1).

\section{al Basic Features.}

It confines particles (mostly electrons)

but releases high energy protons

which interact with the atmosphere

and produce $X$ and $\gamma$ bremsstrahlung.

EG.1. Schematic representation of the plasmoid in the solar magnetic field lines.

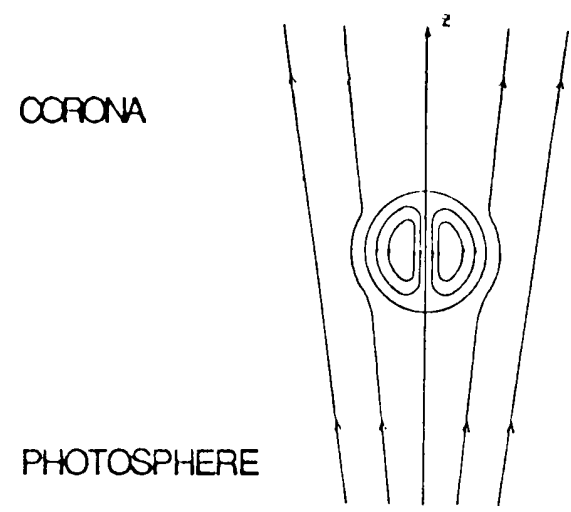

At the top of the atmosphere, the plasmoid explodes and releases the particles recorded in the i.m. Because of the low average density of the plasmoid, confined particles have weakly interacted with matter. With "reasonable" physical parameters (size and density of the plasmoid, internal and external magnetic fields) the ascent time is comparable with the impulsive phase duration (a few 10s). The average density is costimated as $10^{7}-10^{8} \mathrm{~cm}^{-3}$, the instantaneous density decreasing from 
bottom to top of the atmosphere, because of the volume increase.

b/ Dynamics of the plasmoid:

The dynamics of the plasmoid (Raadu et al 1987) can be written as :

$$
M_{c f s} f=D \text { grad }|D|-M n V g+M_{i} n_{i} V_{g}
$$

where Meff is the effective mass of the plasmoid, MnV the mass of volume $V$ with ambient density $n$ and $M i n j V$ the plasmoid mass; $f$ is the acceleration term. The first term on the right-hand side is the magnetic force ( $D$ is the equivalent dipole), the second and third ones are the buoyancy and gravity forces respectively. Neglecting the gravity term (see above) and assuming an exponential law for the field opening (with scale height $H$ ), we arrive at a simple equation relating the velocity of the plasmoid with the initial Alfven velocity and $\mathrm{H}$. Figure 2 shows that an ascent time of about $30 \mathrm{~s}$ can be reached with an Alfven initial velocity of a few $100 \mathrm{~km} / \mathrm{s}$ and a scale height less than $10000 \mathrm{~km}$.

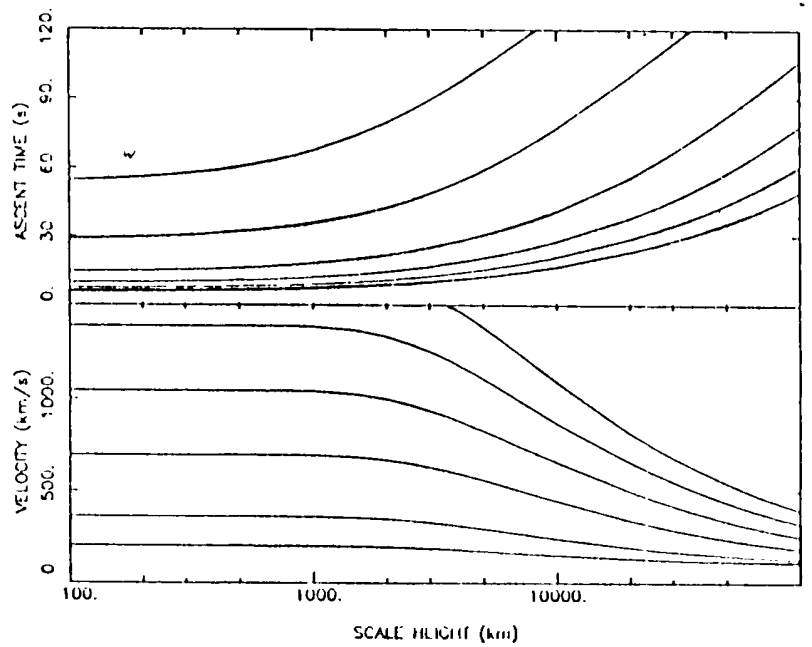

FIG. 2. Variations of the ascent time and the terminal velocity, taken at a height of $10^{4} \mathrm{~km}$, versus the magnetic scale height for different initial Alfven velocities. Alfven velocities have values of $50,100,200,300,400,500 \mathrm{~km} / \mathrm{s}$ for decreasing times (increasing velocities).

\section{CONSEQUENCES OF THE MODEL}

The properties of the plasmoid in relation to the particle transport and release in the solar atmosphere can be summarized as follows:

1/ Protons, nuclei (e. g. 3 He, partially ionized particles) are extracted in the low atmosphere. 2/ They are stored in the magnetic bubble. 3/ They can partially -but gradually- escape in the atmosphere. 4/ Interacting with the atmosphere, particles (especially protons) create $\gamma$ and $X$ rays bremsstrahlung (proton-electron). 5/ Electrons are confined. 6/ Particles left are ejected in the i. m. 7/ Particles have stayed a connstant time in the plasmoid. 8/ Microwave emission results from knock-on electrons. 9/ The $p / e$ ratio may be very variable, as observed (Lin 1973). 


\section{CONCLIUSION}

As far as energetic particles in the i. $m$. are concerned, the proposed model solves the problem of : ${ }^{\circ}$ the chemical and isotopic composition and " the energy spectra. As a result of the censtant lime spent by energetic particles in a low density medium, the traversed matcrial is small. The spectra of energetic particles differ only slightly from a power law. Figure 3 compares the spoctra of electron and proton which have traversed a constant thickness of material or have stayed a constant time in a given medium (plasmoid).

Fig. 3a.

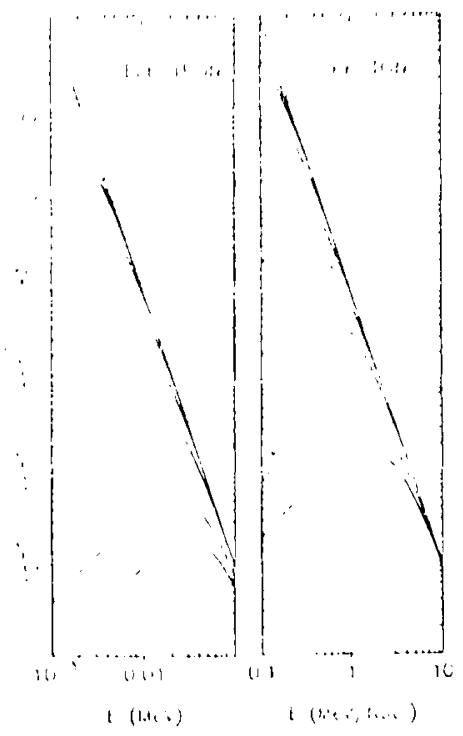

Fig. 3b.

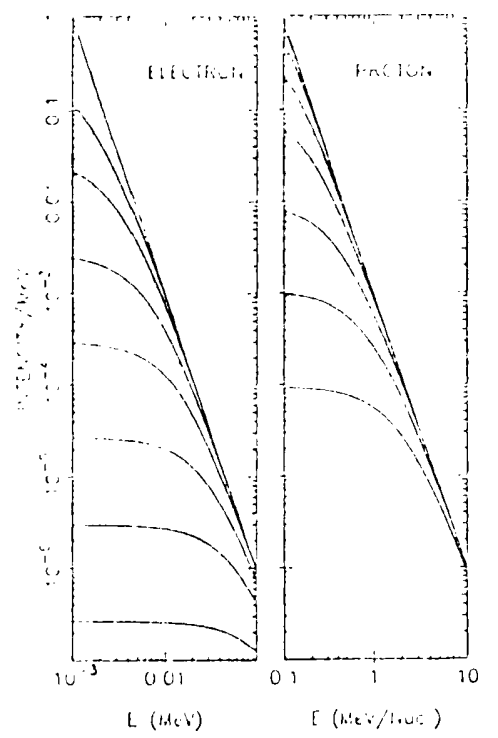

E6.3. Computed spoctra of electrons and protons having traversed a medium of constant thickness $x$ (Fig. $j_{.1}$ ) or having stayed a constant time, given here by the column mass traversed $y$ (Fig. 3b). From top to boltom, $x$ increases from 0 to $10^{-3}\left(50^{-7}, 10^{-6}, 3\right.$ $\left.10^{-6}, 10^{-5}, 310^{-5}, 10^{-4}, 510^{-4}\right)$ and $y$ from 0 to $10^{-2}\left(10^{-5}, 310^{-5}, 10^{-4}, 310^{-4}\right.$, $\left.10 \cdot 3,310^{-3}\right) \mathrm{g} \mathrm{cm}^{2}$.

In the second case (plasmoid), the importance of the traversed material is significantly reduced. This result is u basic feature of our model.

\section{References}

Bryant, D.A., Cline, I.L., Desai, U.D., and McDonald, F.B. 1964, in AASNASA Sympesium on the Physics of Solar Flares, NASA SP-50, p. 289.

Fan, C.Y., Gloocker, G., and llovestadt, D. 1984, Space Sci. Rev., 38, 143. Heristchi et al, 1988, submitted to Ap. J.

Hovestadt, D. et al 1981, Ap. J. (Letters), 246, L81. Kocharov, L.G., and Kocharov, G.E. 1984, Space Sci. Rev., 38, 89.

Lin, R.P. 1973, in Ramaty and Stone (eds.), High Energy Phenomena on the Sun Preceedings, Goddard Space Flight Center, Greenbelt.

Raadu, M.A. et al 1987, Solar Phys., 109, 59.

Ramaty, R., and Murphy, R.J. 1987, Space Sci. Rev., 45, 213.

Svestka, Z. 1976 Selar Flares, D. Reidel Publ. Comp., Dordrecht, Holland. 